\title{
On the Miquel point of simplices
}

\author{
Lothar Heinrich
}

Lothar Heinrich promovierte im Jahr 1980 an der Technischen Universität Dresden. Von 1995-1997 war er Professor für Wahrscheinlichkeitstheorie und Statistik an der Technischen Universität Bergakademie in Freiberg. Seit 1997 ist er Professor für Mathematik an der Universität Augsburg. Seine Forschungsinteressen liegen in der angewandten Wahrscheinlichkeitstheorie (stochastische Geometrie, große Abweichungen, Grenzwertsätze, räumliche Statistik, probabilistische Zahlentheorie).

\section{Introduction and preliminaries}

If points are marked on each side of a planar triangle, one on each side (or on a side's extension), then the three circles (each passing through a vertex and the marked points on the adjacent sides) are concurrent at a point $M$. This interesting fact was first proved and published by Augueste Miquel [3] in 1838, see also Weisstein [4] for further details and extensions. This result is well-known in planar geometry as Miquel's theorem, and $M$ is called the Miquel point. However, much less known (even amongst geometers) is the following multidimensional generalization of Miquel's theorem: If one point is marked on each of the $d(d+1) / 2$ edges of a $d$-simplex

$$
S\left(\mathbf{x}_{\mathbf{0}}, \mathbf{x}_{\mathbf{1}}, \ldots, \mathbf{x}_{\mathbf{d}}\right)=\left\{\mathbf{x}_{\mathbf{0}}+\sum_{i=1}^{d} \mu_{i}\left(\mathbf{x}_{\mathbf{i}}-\mathbf{x}_{\mathbf{0}}\right): \sum_{i=1}^{d} \mu_{i} \leq 1, \mu_{i} \geq 0, i=1, \ldots, d\right\}
$$

Der Satz von Miquel ist ein klassisches Resultat der Elementargeometrie: Wird auf jeder Seite eines gegebenen Dreiecks oder deren Verlängerung ein Punkt beliebig festgelegt, und wird durch jeweils eine Ecke und die beiden markierten Punkte auf den Nachbarseiten ein Kreis gezeichnet, so schneiden sich diese drei Kreise in einem Punkt. In der vorliegenden Arbeit wird mit einfachen Hilfsmitteln der linearen Algebra erstmals ein vollständiger Beweis der Verallgemeinerung des Miquelschen Satzes auf $d$-dimensionale Simplizes angegeben: Wird auf den $d(d+1) / 2$ Kanten je ein Punkt beliebig markiert, dann haben die $d+1$ Kugeln, wovon jede durch je einen Eckpunkt und die markierten Punkte auf den $d$ in diesen Eckpunkt einlaufenden Kanten festgelegt wird, genau einen Punkt gemeinsam. 
with positive volume and a sphere $S_{i}$ is drawn through each vertex $\mathbf{x}_{i}$ and the points marked on the $d$ edges which meet in $\mathbf{x}_{i}$, then these $d+1$ spheres $S_{i}, i=0,1, \ldots, d$, all meet in a point $M$ which will also be called Miquel's point in this note. By the best of the author's knowledge the only known proof of this result seems to be that given by Konnully [2]. Konnully's proof is based on the fact that there exists a common orthogonal sphere with respect to the so-called Miquel spheres $S_{0}, S_{1}, \ldots, S_{d}$ and it is shown that the radius of this sphere equals zero. However, such a sphere does not always exist even in the planar case, namely if the unique point which has the same circle power w.r.t. three pairwise non-concentric circles lies in the interior of each of these circles.

The aim of the present note is to provide a rigorous analytical proof which requires only simple facts from analytic geometry and linear algebra. As a by-product we obtain a family of upper bounds of Gram's determinant (including Hadamard's inequality) which seems to be of interest in its own right. This auxiliary result in Section 3 is valid in all Euclidean vector spaces.

It should be mentioned that an analogous construction with points marked on the $(d-1)$ faces (instead of on the edges) of the simplex does in general not yield a common point that belongs to all spheres.

Let the points of the Euclidean space $\mathbb{R}^{d}$ be represented by column vectors $\mathbf{x}=$ $\left(x_{1}, \ldots, x_{d}\right)^{\prime}$ having the Euclidean norm $\|\mathbf{x}\|=\sqrt{\langle\mathbf{x}, \mathbf{x}\rangle}$, where the scalar product $\langle\cdot, \cdot\rangle$ is defined by

$$
\langle\mathbf{y}, \mathbf{z}\rangle=\mathbf{y}^{\prime} \mathbf{z}=y_{1} z_{1}+\ldots+y_{d} z_{d}
$$

for

$$
\mathbf{y}=\left(y_{1}, \ldots, y_{d}\right)^{\prime} \quad \text { and } \quad \mathbf{z}=\left(z_{1}, \ldots, z_{d}\right)^{\prime}
$$

Furthermore, we recall the well-known fact from analytic geometry that the circumsphere of the $d$-simplex (1) consists of all points $\mathbf{x} \in \mathbb{R}^{d}$ satisfying the equation

$$
\left|\begin{array}{ccccc}
\|\mathbf{x}\|^{2} & \left\|\mathbf{x}_{\mathbf{0}}\right\|^{2} & \left\|\mathbf{x}_{\mathbf{1}}\right\|^{2} & \ldots & \left\|\mathbf{x}_{\mathbf{d}}\right\|^{2} \\
\mathbf{x} & \mathbf{x}_{\mathbf{0}} & \mathbf{x}_{1} & \ldots & \mathbf{x}_{\mathbf{d}} \\
1 & 1 & 1 & \ldots & 1
\end{array}\right|=0
$$

where the left-hand side is for a $(d+2) \times(d+2)$ determinant.

Without loss of generality, we shall assume the vertex $\mathbf{x}_{\mathbf{0}}$ of the $d$-simplex (1) to coincide with the origin $\mathbf{0}=(0, \ldots, 0)^{\prime}$ and the vertices $\mathbf{x}_{\mathbf{i}}=\left(x_{1 i}, \ldots, x_{d i}\right)^{\prime}, i=1, \ldots, d$, to be linearly independent vectors, i.e.

$$
\Delta:=\operatorname{det} \mathbf{X} \neq 0
$$

where $\mathbf{X}=\left(\mathbf{x}_{\mathbf{1}}, \ldots, \mathbf{x}_{\mathbf{d}}\right)$ denotes the quadratic matrix with columns $\mathbf{x}_{\mathbf{1}}, \ldots, \mathbf{x}_{\mathbf{d}}$.

For $0 \leq i<j \leq d$, let $\mathbf{x}_{\mathbf{i j}}$ be a fixed point on the edge joining the vertices $\mathbf{x}_{\mathbf{i}}$ and $\mathbf{x}_{\mathbf{j}}$ being distinct from its end-points, i.e. $\mathbf{x}_{\mathbf{i j}}=\mathbf{x}_{\mathbf{i}}+\lambda_{i j}\left(\mathbf{x}_{\mathbf{j}}-\mathbf{x}_{\mathbf{i}}\right)$ for some $\lambda_{i j} \notin\{0,1\}$. For notational ease put $\lambda_{j i}=1-\lambda_{i j}$ for $i<j$ and $\lambda_{i i}=1 / 2$ for $i=0,1, \ldots, d$. 
In accordance with (2), the unique $d$-sphere $S_{0}$ passing through the origin $\mathbf{o}$ and the points $\mathbf{x}_{\mathbf{0 1}}, \ldots, \mathbf{x}_{\mathbf{0 d}}$ consists of all points $\mathbf{x}=\left(x_{1}, \ldots, x_{d}\right)^{\prime} \in \mathbb{R}^{d}$ satisfying

$$
\Delta_{0}(\mathbf{x}):=\left|\begin{array}{ccccc}
\|\mathbf{x}\|^{2} & 0 & \lambda_{01}^{2}\left\|\mathbf{x}_{\mathbf{1}}\right\|^{2} & \ldots & \lambda_{0 d}^{2}\left\|\mathbf{x}_{\mathbf{d}}\right\|^{2} \\
x_{1} & 0 & \lambda_{01} x_{11} & \ldots & \lambda_{0 d} x_{1 d} \\
\vdots & \vdots & \vdots & \ddots & \vdots \\
x_{d} & 0 & \lambda_{01} x_{d 1} & \ldots & \lambda_{0 d} x_{d d} \\
1 & 1 & 1 & \ldots & 1
\end{array}\right|=0 .
$$

Since $\prod_{i=0}^{d} \lambda_{0 i} \neq 0$, the latter equation is equivalent to

$$
\left|\begin{array}{cccc}
\|\mathbf{x}\|^{2} & \lambda_{01}\left\|\mathbf{x}_{\mathbf{1}}\right\|^{2} & \ldots & \lambda_{0 d}\left\|\mathbf{x}_{\mathbf{d}}\right\|^{2} \\
x_{1} & x_{11} & \ldots & x_{1 d} \\
\vdots & \vdots & \ddots & \vdots \\
x_{d} & x_{d 1} & \ldots & x_{d d}
\end{array}\right|=0 .
$$

Likewise, a point $\mathbf{x}=\left(x_{1}, \ldots, x_{d}\right)^{\prime} \in \mathbb{R}^{d}$ belongs to the unique $d$-sphere $S_{i}$ containing the vertex $\mathbf{x}_{\mathbf{i}}$ and the marked points $\mathbf{x}_{\mathbf{0} \mathbf{i}}, \ldots, \mathbf{x}_{\mathbf{i}-\mathbf{1}, \mathbf{i}}, \mathbf{x}_{\mathbf{i}, \mathbf{i}+\mathbf{1}}, \ldots, \mathbf{x}_{\mathbf{i d}}$ on the adjacent edges if and only if

$$
\Delta_{i}(\mathbf{x}):=\left|\begin{array}{cccc}
\|\mathbf{x}\|^{2} & \left\|\mathbf{x}_{\mathbf{i}}\right\|^{2} & \lambda_{0 i}^{2}\left\|\mathbf{x}_{\mathbf{i}}\right\|^{2} & \left\|\mathbf{x}_{\mathbf{i}}+\lambda_{i j}\left(\mathbf{x}_{\mathbf{j}}-\mathbf{x}_{\mathbf{i}}\right)\right\|^{2} \\
x_{1} & x_{1 i} & \lambda_{0 i} x_{1 i} & x_{1 i}+\lambda_{i j}\left(x_{1 j}-x_{1 i}\right) \\
\vdots & \vdots & \vdots & \vdots \\
x_{d} & x_{d i} & \lambda_{0 i} x_{d i} & x_{d i}+\lambda_{i j}\left(x_{d j}-x_{d i}\right) \\
1 & 1 & 1 & \underbrace{1}_{j \in\{1, \ldots, d\} \backslash\{i\}}
\end{array}\right|=0
$$

for $i=1, \ldots, d$.

By appealing to the well-known transformation rules for determinants we obtain

$$
\begin{aligned}
& \Delta_{i}(\mathbf{x})=\left(\lambda_{0 i}-1\right)\left|\begin{array}{cccc}
\|\mathbf{x}\|^{2}-\lambda_{0 i}^{2}\left\|\mathbf{x}_{\mathbf{i}}\right\|^{2} & \left(1+\lambda_{0 i}\right)\left\|\mathbf{x}_{\mathbf{i}}\right\|^{2} & \lambda_{0 i}\left\|\mathbf{x}_{\mathbf{i}}\right\|^{2} & \left\|\mathbf{x}_{\mathbf{i}}+\lambda_{i j}\left(\mathbf{x}_{\mathbf{j}}-\mathbf{x}_{\mathbf{i}}\right)\right\|^{2} \\
x_{1}-\lambda_{0 i} x_{1 i} & x_{1 i} & 0 & x_{1 i}+\lambda_{i j}\left(x_{1 j}-x_{1 i}\right) \\
\vdots & \vdots & \vdots & \vdots \\
x_{d}-\lambda_{0 i} x_{d i} & x_{d i} & 0 & x_{d i}+\lambda_{i j}\left(x_{d j}-x_{d i}\right) \\
0 & 0 & -1 & \underbrace{1}_{j \in\{1, \ldots, d\} \backslash\{i\}}
\end{array}\right| \\
& =\left(\lambda_{0 i}-1\right) \prod_{j \neq i} \lambda_{i j} \mid \begin{array}{cccc}
\|\mathbf{x}\|^{2}-\lambda_{0 i}^{2}\left\|\mathbf{x}_{\mathbf{i}}\right\|^{2} & c_{i i} & \lambda_{0 i}\left\|\mathbf{x}_{\mathbf{i}}\right\|^{2} & c_{i j} \\
x_{1}-\lambda_{0 i} x_{1 i} & x_{1 i} & 0 & x_{1 j} \\
\vdots & \vdots & \vdots & \vdots \\
x_{d}-\lambda_{0 i} x_{d i} & x_{d i} & 0 & x_{d j} \\
0 & 0 & -1 & \underbrace{0}_{j \in\{1, \ldots, d\} \backslash\{i\}}
\end{array},
\end{aligned}
$$


where

$$
\begin{aligned}
c_{i j} & :=\frac{\left\|\mathbf{x}_{\mathbf{i}}+\lambda_{i j}\left(\mathbf{x}_{\mathbf{j}}-\mathbf{x}_{\mathbf{i}}\right)\right\|^{2}-\left\|\mathbf{x}_{\mathbf{i}}\right\|^{2}}{\lambda_{i j}}+\left(1+\lambda_{0 i}\right)\left\|\mathbf{x}_{\mathbf{i}}\right\|^{2} \\
& =\lambda_{0 i}\left\|\mathbf{x}_{\mathbf{i}}\right\|^{2}+\left\|\mathbf{x}_{\mathbf{j}}\right\|^{2}-\lambda_{j i}\left\|\mathbf{x}_{\mathbf{i}}-\mathbf{x}_{\mathbf{j}}\right\|^{2} \text { for } i, j=1, \ldots, d .
\end{aligned}
$$

The latter equality follows by using the identity $\|\mathbf{u}-\mathbf{v}\|^{2}=\|\mathbf{u}\|^{2}+\|\mathbf{v}\|^{2}-2\langle\mathbf{u}, \mathbf{v}\rangle$. Since $c_{i i}=\left(1+\lambda_{0 i}\right)\left\|\mathbf{x}_{\mathbf{i}}\right\|^{2}$ we may simplify the previous determinant by multiplying the second column by $\lambda_{0 i}$ and adding it to the first column.

Consequently, in view of $\lambda_{i j} \notin\{0,1\}$ for $i \neq j$, the equation $\Delta_{i}(\mathbf{x})=0$ can be expressed as

$$
\left|\begin{array}{cccc}
\|\mathbf{x}\|^{2}+\lambda_{0 i}\left\|\mathbf{x}_{\mathbf{i}}\right\|^{2} & c_{i 1} & \cdots & c_{i d} \\
x_{1} & x_{11} & \cdots & x_{1 d} \\
\vdots & \vdots & \ddots & \vdots \\
x_{d} & x_{d 1} & \cdots & x_{d d}
\end{array}\right|=0 \text { for } i=1, \ldots, d .
$$

Obviously, the set of points $\mathbf{x} \in \mathbb{R}^{d}$ satisfying both equations (4) and (5) coincides with the $(d-1)$-dimensional sphere $S_{0} \cap S_{i}$. By subtracting equation (4) from equation (5) and applying the summation law for determinants differing in only one row we obtain the linear equation

$$
D_{i}(\mathbf{x}):=\left|\begin{array}{cccc}
\lambda_{0 i}\left\|\mathbf{x}_{\mathbf{i}}\right\|^{2} & c_{i 1}-\lambda_{01}\left\|\mathbf{x}_{\mathbf{1}}\right\|^{2} & \cdots & c_{i d}-\lambda_{0 d}\left\|\mathbf{x}_{\mathbf{d}}\right\|^{2} \\
x_{1} & x_{11} & \cdots & x_{1 d} \\
\vdots & \vdots & \ddots & \vdots \\
x_{d} & x_{d 1} & \cdots & x_{d d}
\end{array}\right|=0
$$

that holds for all $\mathbf{x}=\left(x_{1}, \ldots, x_{d}\right)^{\prime} \in \mathbb{R}^{d}$ belonging to the (uniquely determined) hyperplane $H_{i}$ containing the $(d-1)$-sphere $S_{0} \cap S_{i}$. Combining the equations $D_{i}(\mathbf{x})=0$, $i=1, \ldots, d$, yields a system of $d$ linear equations whose solution (if it exists!) coincides with the point of intersection of the hyperplanes $H_{1}, \ldots, H_{d}$.

To find this point we introduce the matrix $\mathbf{A}_{\Lambda}=\left(a_{i j}\right)_{i, j=1}^{d}$ with entries

$$
a_{i j}:=c_{i j}-\lambda_{0 j}\left\|\mathbf{x}_{\mathbf{j}}\right\|^{2}=\lambda_{0 i}\left\|\mathbf{x}_{\mathbf{i}}\right\|^{2}+\left(1-\lambda_{0 j}\right)\left\|\mathbf{x}_{\mathbf{j}}\right\|^{2}-\lambda_{j i}\left\|\mathbf{x}_{\mathbf{i}}-\mathbf{x}_{\mathbf{j}}\right\|^{2} .
$$

Let us first note the remarkable fact that, in view of $\lambda_{i j}+\lambda_{j i}=1$ for $i \neq j$ and $\lambda_{i i}=1 / 2$, $a_{i j}+a_{j i}=\left\|\mathbf{x}_{\mathbf{i}}\right\|^{2}+\left\|\mathbf{x}_{\mathbf{j}}\right\|^{2}-\left(\lambda_{i j}+\lambda_{j i}\right)\left\|\mathbf{x}_{\mathbf{i}}-\mathbf{x}_{\mathbf{j}}\right\|^{2}=2\left\langle\mathbf{x}_{\mathbf{i}}, \mathbf{x}_{\mathbf{j}}\right\rangle$ for $i, j=1, \ldots, d$, which can be expressed concisely by

$$
\frac{1}{2}\left(\mathbf{A}_{\Lambda}+\mathbf{A}_{\Lambda}^{\prime}\right)=G(\mathbf{X}):=\left(\left\langle\mathbf{x}_{\mathbf{i}}, \mathbf{x}_{\mathbf{j}}\right\rangle\right)_{i, j=1}^{d}
$$

where $G(\mathbf{X})$ and det $G(\mathbf{X})$ are called Gram's matrix and Gram's determinant, respectively, of the vectors $\mathbf{x}_{\mathbf{1}}, \ldots, \mathbf{x}_{\mathbf{d}}$. In $\mathbb{R}^{d}$ we have $G(\mathbf{X})=\mathbf{X}^{\prime} \mathbf{X}$ and thus $\operatorname{det} G(\mathbf{X})=\Delta^{2}$. On the 
other hand, the relation (7) is meaningful in any real vector space $V$ on which a symmetric, positive definite, bilinear form $\langle\cdot, \cdot\rangle$ - briefly called scalar product - is defined. Such vector spaces are usually called Euclidean vector spaces.

Proposition. For arbitrary linearly independent vectors $\mathbf{x}_{\mathbf{1}}, \ldots, \mathbf{x}_{\mathbf{d}} \in \mathbb{R}^{d}$ and real numbers $\lambda_{i j}, i, j=0,1, \ldots, d$, satisfying $\lambda_{i j}+\lambda_{j i}=1$ and $\lambda_{i j} \notin\{0,1\}$ for $i, j=0,1, \ldots, d$, there exists a unique point of intersection $H_{1} \cap \ldots \cap H_{d}=\mathbf{x}^{*}=\left(x_{1}^{*}, \ldots, x_{d}^{*}\right)^{\prime}$, which is given by

$$
\mathbf{x}^{*}=\mathbf{X} \mathbf{A}_{\Lambda}^{-1} \mathbf{x}_{\lambda} \quad \text { with } \quad \mathbf{x}_{\lambda}=\left(\lambda_{01}\left\|\mathbf{x}_{\mathbf{1}}\right\|^{2}, \ldots, \lambda_{0 d}\left\|\mathbf{x}_{\mathbf{d}}\right\|^{2}\right)^{\prime}
$$

The first step in proving this result is to show that $\mathbf{x}=\left(x_{1}, \ldots, x_{d}\right)^{\prime}$ obeys the linear equations $D_{i}(\mathbf{x})=0$ for $i=1, \ldots, d$ if and only if it satisfies the equation $\mathbf{A}_{\Lambda} \mathbf{X}^{-1} \mathbf{x}=$ $\mathbf{x}_{\lambda}$. This is left to the reader as an exercise. To see the invertibility of the matrix $\mathbf{A}_{\Lambda}$ we use (7) and decompose $\mathbf{A}_{\Lambda}$ as follows:

$$
\mathbf{A}_{\Lambda}=\mathbf{B}_{\Lambda}+G(\mathbf{X}) \quad \text { with } \quad \mathbf{B}_{\Lambda}=\frac{1}{2}\left(\mathbf{A}_{\Lambda}-\mathbf{A}_{\Lambda}^{\prime}\right)
$$

Here the matrix $\mathbf{B}_{\Lambda}$ is skew-symmetric, i.e. $\mathbf{B}_{\Lambda}^{\prime}=-\mathbf{B}_{\Lambda}$. This skew-symmetry and the positive definiteness of the Gram matrix $G(\mathbf{X})$ show the quadratic form $\mathbf{x}^{\prime} \mathbf{A}_{\Lambda} \mathbf{x}=$ $\mathbf{x}^{\prime} G(\mathbf{X}) \mathbf{x}$ to be strictly positive for any $\mathbf{x} \neq \mathbf{o}$. This in turn implies $\mathbf{A}_{\Lambda} \mathbf{x} \neq \mathbf{o}$ for any $\mathbf{x} \neq$ $\mathbf{o}$, which is equivalent to $\operatorname{det} \mathbf{A}_{\Lambda} \neq 0$ for all $\lambda_{i j}$ satisfying $\lambda_{i j}+\lambda_{j i}=1$. This combined with a simple continuity argument tells us that $\operatorname{det} \mathbf{A}_{\Lambda}<0$ contradicts $\operatorname{det} G(\mathbf{X})>0$, leaving as the only possibility $\operatorname{det} \mathbf{A}_{\Lambda}>0$. By the same $\operatorname{argument}$ we $g e t \operatorname{det}\left(\mathbf{B}_{\Lambda}+\right.$ $\alpha G(\mathbf{X}))>0$ for any $\alpha>0$, entailing $\operatorname{det} \mathbf{B}_{\Lambda} \geq 0$ by letting $\alpha \downarrow 0$. However $\operatorname{det} \mathbf{B}_{\Lambda}$ may take positive values only for even $d \geq 2$ due to the very definition of skew-symmetry. Moreover, we shall bound $\operatorname{det} \mathbf{A}_{\Lambda}$ uniformly from below by Gram's determinant $\operatorname{det} G(\mathbf{X})$ in Section 3.

Finally, note that the proposition does not answer the question whether $\mathbf{x}^{*} \in S_{i}$ holds for some or even all $i=0,1, \ldots, d$. This will be the subject of the next section.

\section{The main result and its proof}

Theorem. Under the conditions of the above proposition the point $\mathbf{x}^{*}$ given in (8) belongs to each of the spheres $S_{i}, i=0,1, \ldots, d$, that is, $\mathbf{x}^{*}$ is the unique point of intersection $S_{0} \cap S_{1} \cap \ldots \cap S_{d}$. In other words, $\mathbf{x}^{*}$ coincides with Miquel's point $M$ of the simplex $S\left(\mathbf{o}, \mathbf{x}_{\mathbf{1}}, \ldots, \mathbf{x}_{\mathbf{d}}\right)$ w.r.t. the marked points $\mathbf{x}_{\mathbf{i j}}=\mathbf{x}_{\mathbf{i}}+\lambda_{i j}\left(\mathbf{x}_{\mathbf{j}}-\mathbf{x}_{\mathbf{i}}\right)$ on its edges.

Proof. After transposing and expanding the determinant on the left-hand side of (4) along the first row we recognize that $\Delta_{0}\left(\mathbf{x}^{*}\right)=0$ is equivalent to

$$
\left\|\mathbf{x}^{*}\right\|^{2} \Delta-\sum_{j=1}^{d} x_{j}^{*}\left|\begin{array}{ccccc}
x_{11} & \cdots & \lambda_{01}\left\|\mathbf{x}_{\mathbf{1}}\right\|^{2} & \cdots & x_{d 1} \\
x_{12} & \cdots & \lambda_{02}\left\|\mathbf{x}_{\mathbf{2}}\right\|^{2} & \cdots & x_{d 2} \\
\vdots & & \vdots & & \vdots \\
x_{1 d} & \cdots & \lambda_{0 d}\left\|\mathbf{x}_{\mathbf{d}}\right\|^{2} & \cdots & x_{d d}
\end{array}\right|=0 .
$$




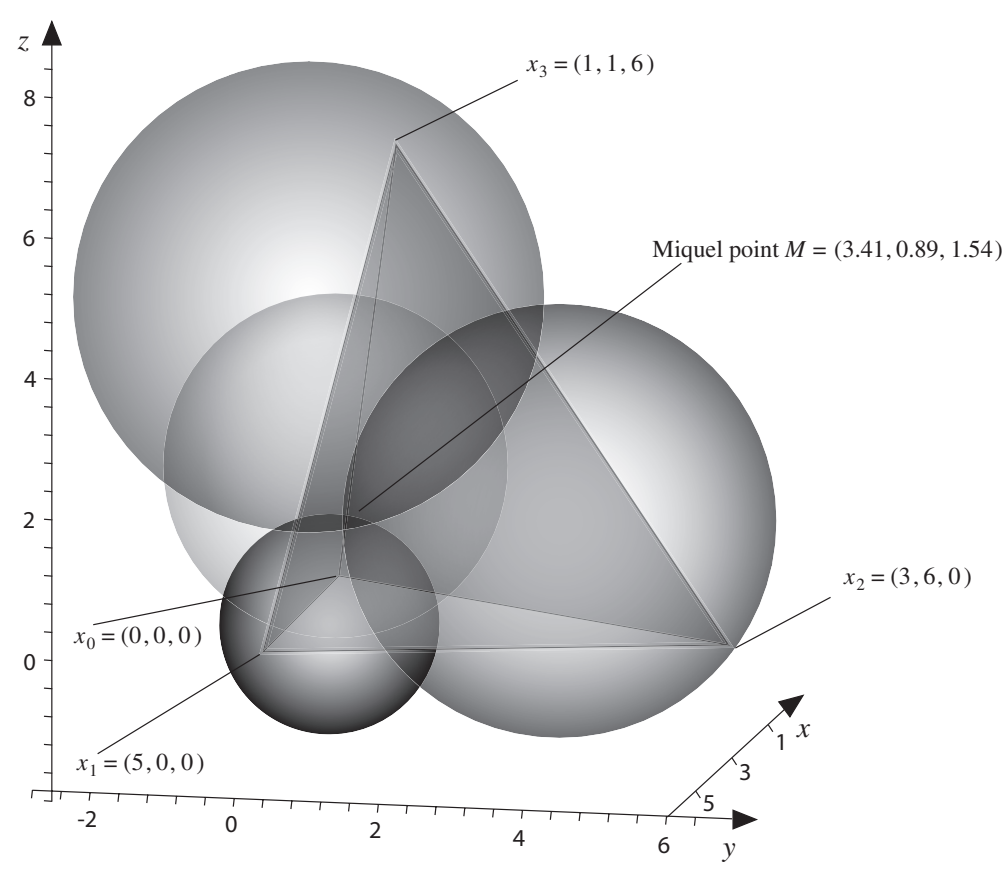

Fig. 1: Tetrahedron $S\left(\mathbf{x}_{\mathbf{0}}, \mathbf{x}_{\mathbf{1}}, \mathbf{x}_{\mathbf{2}}, \mathbf{x}_{\mathbf{3}}\right)$ and the four Miquel spheres with common Miquel point $M$ for $\lambda_{01}=1 / 2, \lambda_{02}=1 / 4, \lambda_{03}=2 / 3, \lambda_{12}=1 / 3, \lambda_{13}=$ $1 / 4, \lambda_{23}=2 / 3$

Dividing by $\Delta \neq 0$ the latter equation takes the form $\left\langle\mathbf{x}^{*}, \mathbf{x}^{*}-\mathbf{z}_{\mathbf{0}}\right\rangle=0$, where the components of $\mathbf{z}_{\mathbf{0}}=\left(z_{1}, \ldots, z_{d}\right)^{\prime}$ are given by

$$
z_{j}=\frac{1}{\Delta}\left|\begin{array}{ccccc}
x_{11} & \cdots & \lambda_{01}\left\|\mathbf{x}_{\mathbf{1}}\right\|^{2} & \cdots & x_{d 1} \\
x_{12} & \cdots & \lambda_{02}\left\|\mathbf{x}_{\mathbf{2}}\right\|^{2} & \cdots & x_{d 2} \\
\vdots & & \vdots & & \vdots \\
x_{1 d} & \cdots & \lambda_{0 d}\left\|\mathbf{x}_{\mathbf{d}}\right\|^{2} & \cdots & x_{d d}
\end{array}\right| \quad \text { for } \quad j=1, \ldots, d
$$

Applying Cramer's rule we see that $\mathbf{z}_{\mathbf{0}}$ satisfies the linear equation $\mathbf{X}^{\prime} \mathbf{z}_{\mathbf{0}}=\mathbf{x}_{\lambda}$, i.e. $\left\langle\mathbf{x}_{\mathbf{i}} /\left\|\mathbf{x}_{\mathbf{i}}\right\|, \mathbf{z}_{\mathbf{0}}\right\rangle=\lambda_{0 i}\left\|\mathbf{x}_{\mathbf{i}}\right\|$ for $i=1, \ldots, d$, so that $\mathbf{z}_{\mathbf{0}}=\left(\mathbf{X}^{\prime}\right)^{-1} \mathbf{x}_{\lambda}$. The geometric interpretation of the above relations reveals that the orthogonal projection of $\mathbf{z}_{\mathbf{0}}$ onto the edge joining $\mathbf{o}$ and $\mathbf{x}_{\mathbf{i}}$ equals $\mathbf{x}_{\mathbf{0}}=\lambda_{0 i} \mathbf{x}_{\mathbf{i}}$ for $i=1, \ldots, d$. Furthermore, $\mathbf{z}_{\mathbf{0}}$ lies on the sphere $S_{0}$ and, provided that $\mathbf{x}^{*} \in S_{0}$, the point $\mathbf{z}_{\mathbf{0}} / 2$ coincides with the centre of $S_{0}$ by appealing to the converse of Thales' theorem.

Hence, $\Delta_{0}\left(\mathbf{x}^{*}\right)=0$ holds if and only if

$$
\left\langle\mathbf{x}^{*}, \mathbf{x}^{*}-\left(\mathbf{X}^{\prime}\right)^{-1} \mathbf{x}_{\lambda}\right\rangle=\mathbf{x}_{\lambda}^{\prime}\left(\mathbf{A}_{\Lambda}^{-1}\right)^{\prime} \mathbf{X}^{\prime}\left(\mathbf{X} \mathbf{A}_{\Lambda}^{-1}-\left(\mathbf{X}^{\prime}\right)^{-1}\right) \mathbf{x}_{\lambda}=\mathbf{x}_{\lambda}^{\prime} \mathbf{C}_{\Lambda} \mathbf{x}_{\lambda}=0,
$$

where $\mathbf{C}_{\Lambda}:=\left(\mathbf{A}_{\Lambda}^{-1}\right)^{\prime} \mathbf{X}^{\prime} \mathbf{X} \mathbf{A}_{\Lambda}^{-1}-\left(\mathbf{A}_{\Lambda}^{-1}\right)^{\prime}$ 


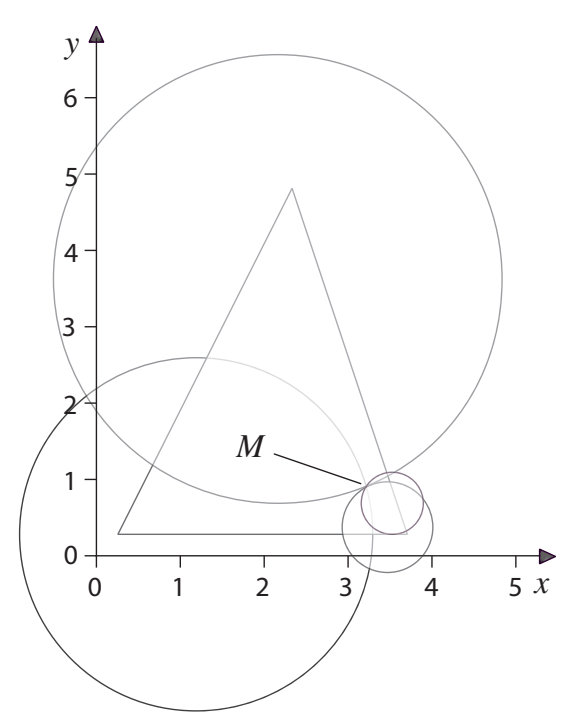

Fig. 2: Intersection of the tetrahedron and the Miquel spheres in Fig. 1 with the plane $z=1.54$ parallel to the $x y$-plane

Using (7) we find that

$$
\mathbf{C}_{\Lambda}=\frac{1}{2}\left(\mathbf{A}_{\Lambda}^{-1}\right)^{\prime}\left(\mathbf{A}_{\Lambda}+\mathbf{A}_{\Lambda}^{\prime}\right) \mathbf{A}_{\Lambda}^{-1}-\left(\mathbf{A}_{\Lambda}^{-1}\right)^{\prime}=\frac{1}{2}\left(\mathbf{A}_{\Lambda}^{-1}-\left(\mathbf{A}_{\Lambda}^{-1}\right)^{\prime}\right)=-\mathbf{C}_{\Lambda}^{\prime},
$$

thus proving the skew-symmetry of the matrix $\mathbf{C}_{\Lambda}$ which is necessary and sufficient for the quadratic form $\mathbf{x}_{\lambda}^{\prime} \mathbf{C}_{\Lambda} \mathbf{x}_{\lambda}$ to disappear for any real $\lambda_{01}, \ldots, \lambda_{0 d}$ and any $\mathbf{x}_{\mathbf{1}}, \ldots, \mathbf{x}_{\mathbf{d}} \in \mathbb{R}^{d}$, as we wished to prove.

It remains to show that $\Delta_{i}\left(\mathbf{x}^{*}\right)=0$ for $i=1, \ldots, d$. For this we transpose the determinant on the left-hand side of (5) and expand it along the first row leading to the system of equations

$$
\left(\left\|\mathbf{x}^{*}\right\|^{2}+\lambda_{0 i}\left\|\mathbf{x}_{\mathbf{i}}\right\|^{2}\right) \Delta-\sum_{j=1}^{d} x_{j}^{*}\left|\begin{array}{ccccc}
x_{11} & \cdots & c_{i 1} & \cdots & x_{d 1} \\
\vdots & & \vdots & & \vdots \\
x_{1 d} & \cdots & \underbrace{c_{i d}}_{j \text {-th column }} & \cdots & x_{d d}
\end{array}\right|=0, i=1, \ldots, d .
$$

In the next step we subtract equation (9) from the latter equation and divide the difference by $\Delta \neq 0$. Finally, using the abbreviation (6) we arrive at the equation

$$
\lambda_{0 i}\left\|\mathbf{x}_{\mathbf{i}}\right\|^{2}=\sum_{j=1}^{d} x_{j}^{*} w_{i j} \text { with } w_{i j}=\frac{1}{\Delta}\left|\begin{array}{ccccc}
x_{11} & \cdots & a_{i 1} & \cdots & x_{d 1} \\
\vdots & & \vdots & & \vdots \\
x_{1 d} & \cdots & \underbrace{a_{i d}}_{j \text {-th column }} & \cdots & x_{d d}
\end{array}\right|, j=1, \ldots, d,
$$


which is equivalent to $\Delta_{i}\left(\mathbf{x}^{*}\right)=0$ for each $i=1, \ldots, d$. Again, according to Cramer's rule the vector $\mathbf{w}_{\mathbf{i}}=\left(w_{i 1}, \ldots, w_{i d}\right)^{\prime}$ satisfies the equation $\mathbf{X}^{\prime} \mathbf{w}_{\mathbf{i}}=\left(a_{i 1}, \ldots, a_{i d}\right)^{\prime}$, that is, we may write $\mathbf{w}_{\mathbf{i}}=\left(\mathbf{X}^{\prime}\right)^{-1} \mathbf{A}_{\Lambda}^{\prime} \mathbf{e}_{\mathbf{i}}$ with $\mathbf{e}_{\mathbf{i}}=(0, \ldots, 1, \ldots, 0)^{\prime}$ denoting the $i$-th column vector of the $d \times d$ identity matrix. Hence, the above equations can be rewritten as

$$
\lambda_{0 i}\left\|\mathbf{x}_{\mathbf{i}}\right\|^{2}=\mathbf{w}_{\mathbf{i}}^{\prime} \mathbf{x}^{*}=\mathbf{e}_{\mathbf{i}}^{\prime} \mathbf{A}_{\Lambda} \mathbf{X}^{-1} \mathbf{x}^{*} \text { for } i=1, \ldots, d .
$$

However, these equations follow directly from (8) and vice versa. Thus the proof of the Theorem is complete.

Note that the point $\mathbf{z}_{\mathbf{i}}=\mathbf{w}_{\mathbf{i}}+\mathbf{z}_{\mathbf{0}}-\mathbf{x}_{\mathbf{i}}$ which can be shown to belong to the sphere $S_{i}$ satisfies the orthogonality relation

$$
\left\langle\mathbf{x}^{*}-\mathbf{z}_{\mathbf{i}}, \mathbf{x}^{*}-\mathbf{x}_{\mathbf{i}}\right\rangle=0 \text { for } i=1, \ldots, d .
$$

This is easily verified by straightforward computations using the expressions of $\mathbf{x}^{*}, \mathbf{w}_{\mathbf{i}}$, and $\mathbf{z}_{\mathbf{0}}$ given in the above proof. As a result, the sphere $S_{i}$ has the centre $\left(\mathbf{z}_{\mathbf{i}}+\mathbf{x}_{\mathbf{i}}\right) / 2=$ $\left(\mathbf{w}_{\mathbf{i}}+\mathbf{z}_{\mathbf{0}}\right) / 2$.

\section{Bounds for determinants}

In the subsequent lemma we establish a lower bound for the determinant of $\mathbf{A}_{\Lambda}$ that is uniform in all the varying parameters $\lambda_{i j}, 0 \leq i<j \leq d$, and even positive provided the vectors $\mathbf{x}_{\mathbf{1}}, \ldots, \mathbf{x}_{\mathbf{d}}$ are linearly independent. Note that the below inequality (11) is valid for any $d(\geq 2)$ elements of an arbitrary Euclidean vector space.

Lemma. Let $V$ be a Euclidean vector space equipped with scalar product $\langle\cdot, \cdot\rangle$. For any $\mathbf{x}_{\mathbf{1}}, \ldots, \mathbf{x}_{\mathbf{d}} \in V$ and any real numbers $\lambda_{i j}, i, j=0,1, \ldots, d$, satisfying $\lambda_{i j}+\lambda_{j i}=1$ for $i, j=0,1, \ldots, d$, the inequality

$$
\operatorname{det} \mathbf{A}_{\Lambda} \geq \operatorname{det} \mathbf{B}_{\Lambda}+\operatorname{det} G(\mathbf{X}) \geq \operatorname{det} G(\mathbf{X})
$$

holds, where $\mathbf{A}_{\Lambda}, \mathbf{B}_{\Lambda}$, and $G(\mathbf{X})$ are defined by (6), (9), and (7), respectively.

Equality is attained in (11) if

$$
\lambda_{i j}\left\|\mathbf{x}_{\mathbf{i}}-\mathbf{x}_{\mathbf{j}}\right\|^{2}=\left(1-\lambda_{0 i}\right)\left\|\mathbf{x}_{\mathbf{i}}\right\|^{2}+\lambda_{0 j}\left\|\mathbf{x}_{\mathbf{j}}\right\|^{2}-\left\langle\mathbf{x}_{\mathbf{i}}, \mathbf{x}_{\mathbf{j}}\right\rangle \quad \text { for } \quad 1 \leq i<j \leq d .
$$

Proof. As already pointed out at the end of Section 1 the determinant of any skewsymmetric $d \times d$ matrix $\mathbf{B}$ is non-negative and equals zero if $d$ is odd. Thus, the second part of (11) is trivial and instead of the first part we prove the slightly more general inequality

$$
\operatorname{det}(\mathbf{B}+G(\mathbf{X})) \geq \operatorname{det} \mathbf{B}+\operatorname{det} G(\mathbf{X}) .
$$

By applying the well-known principal axis theorem to the non-negative definite Gram matrix $G(\mathbf{X})$ we find an orthogonal $d \times d$ matrix $\mathbf{O}$ (with $\operatorname{det} \mathbf{O}=1$ ) such that $\mathbf{D}=$ $\mathbf{O}^{\prime} G(\mathbf{X}) \mathbf{O}$ is a diagonal matrix with non-negative diagonal elements. The multiplication rule for determinants enables us to replace $G(\mathbf{X})$ by $\mathbf{D}$ and $\mathbf{B}$ by the skew-symmetric matrix 
$\mathbf{O}^{\prime} \mathbf{B} \mathbf{O}$ without changing the inequality (12). Therefore it suffices to verify $\operatorname{det}(\mathbf{B}+\mathbf{D}) \geq$ $\operatorname{det} \mathbf{B}+\operatorname{det} \mathbf{D}$ only for diagonal matrices $\mathbf{D}$ with non-negative diagonal elements $y_{1}, \ldots, y_{d}$ and any skew-symmetric $d \times d$ matrix $\mathbf{B}$. The Taylor expansion of the function

$$
f\left(y_{1}, \ldots, y_{d}\right)=\operatorname{det}(\mathbf{B}+\mathbf{D})=\left|\begin{array}{cccc}
y_{1} & b_{12} & \cdots & b_{1 d} \\
-b_{12} & y_{2} & \cdots & b_{2 d} \\
\vdots & \vdots & & \vdots \\
-b_{1 d} & -b_{2 d} & \cdots & y_{d}
\end{array}\right|
$$

leads to

$$
f\left(y_{1}, \ldots, y_{d}\right)=\operatorname{det} \mathbf{B}+\sum_{k=1}^{d-2} \sum_{1 \leq i_{1}<\cdots<i_{k} \leq d} y_{i_{1}} \cdots y_{i_{k}} \operatorname{det} \mathbf{B}_{\mathbf{i}_{1}, \ldots, \mathbf{i}_{\mathbf{k}}}+y_{1} \cdots y_{d},
$$

where the $(d-k) \times(d-k)$ matrix $\mathbf{B}_{\mathbf{i}_{1}, \ldots, \mathbf{i}_{\mathbf{k}}}$ emerges from $\mathbf{B}$ by deleting the rows $i_{1}, \ldots, i_{k}$ and columns $i_{1}, \ldots, i_{k}$. Obviously, all these matrices are skew-symmetric which, together with $y_{1}, \ldots, y_{d} \geq 0$, implies

$$
f\left(y_{1}, \ldots, y_{d}\right) \geq \operatorname{det} \mathbf{B}+y_{1} \cdots y_{d}=\operatorname{det} \mathbf{B}+\operatorname{det} \mathbf{D} .
$$

Thus the inequality (11) is proved. The proof of the lemma is complete by noting that $\mathbf{A}_{\Lambda}=\mathbf{A}_{\Lambda}^{\prime}$ and (7) imply equality in (11).

Remark. The above proof turns out that the inequality (12) remains valid if $G(\mathbf{X})$ is replaced by any other non-negative definite $d \times d$ matrix.

Corollary. If $\mathbf{x}_{1}, \ldots, \mathbf{x}_{\mathbf{d}} \in V$ are linearly independent, then $\operatorname{det} \mathbf{A}_{\Lambda}$ is positive which in turn implies the existence of the inverse $\mathbf{A}_{\Lambda}^{-1}$ for all real $\lambda_{i j}$ satisfying $\lambda_{i j}+\lambda_{j i}=1$ for $i, j=0,1, \ldots, d$. As a special case, (11) includes the well-known Hadamard inequality

$$
\operatorname{det} G(\mathbf{X}) \leq\left\|\mathbf{x}_{\mathbf{1}}\right\|^{2} \cdot \ldots \cdot\left\|\mathbf{x}_{\mathbf{d}}\right\|^{2} \quad \text { for any } \quad x_{1}, \ldots, x_{d} \in V,
$$

which reads $|\Delta| \leq\left\|\mathbf{x}_{\mathbf{1}}\right\| \cdot \ldots \cdot\left\|\mathbf{x}_{\mathbf{d}}\right\| \quad$ in $\mathbb{R}^{d}$.

The first part of the corollary follows from the well-known fact that $\operatorname{det} G(\mathbf{X})>0$ characterizes the linear independence of $x_{1}, \ldots, x_{d} \in V$. The second part follows by setting $\lambda_{01}=\ldots=\lambda_{0 d}=0$ and $\lambda_{i j}=\left\|\mathbf{x}_{\mathbf{i}}\right\|^{2} /\left\|\mathbf{x}_{\mathbf{i}}-\mathbf{x}_{\mathbf{j}}\right\|^{2}$ so that by (6) $a_{j i}=0$ for $1 \leq i<j \leq d$. In other words, $\mathbf{A}_{\Lambda}$ is an upper triangular matrix entailing $\operatorname{det} \mathbf{A}_{\Lambda}=a_{11} \cdot \ldots \cdot a_{d d}$.

\section{Concluding remarks}

1. Formula (8) and the above theorem reveal that the Miquel point $M$ of the simplex $S\left(\mathbf{o}, \mathbf{x}_{\mathbf{1}}, \ldots, \mathbf{x}_{\mathbf{d}}\right)$ w.r.t. the marked points $\mathbf{x}_{\mathbf{i j}}=\mathbf{x}_{\mathbf{i}}+\lambda_{i j}\left(\mathbf{x}_{\mathbf{j}}-\mathbf{x}_{\mathbf{i}}\right)$ on the simplex' edges can be represented as a linear combination of the edges $\mathbf{x}_{\mathbf{1}}, \ldots, \mathbf{x}_{\mathbf{d}}$,

$$
\mathbf{x}^{*}=\sum_{i=1}^{d} u_{i} \mathbf{x}_{\mathbf{i}}=\mathbf{X} \mathbf{u} \quad \text { with weight vector } \quad \mathbf{u}=\left(u_{1}, \ldots, u_{d}\right)^{\prime}=\mathbf{A}_{\Lambda}^{-1} \mathbf{x}_{\lambda} .
$$


From (6) and (8) it is seen that the weights $u_{1}, \ldots, u_{d}$ depend only on the parameters $\lambda_{i j}$ and the squared edge lengths $\left\|\mathbf{x}_{\mathbf{i}}-\mathbf{x}_{\mathbf{j}}\right\|^{2}$ for $0 \leq i<j \leq d$ (with $\mathbf{x}_{\mathbf{0}}=\mathbf{0}$ ). For this reason the Miquel point can be defined in a meaningful way for any finite family of linearly independent elements of a Euclidean vector space $V$.

By (13) the Miquel points $\mathbf{x}_{\mathbf{i}}^{*}, i=0,1, \ldots, d$, of those $(d-1)$-simplices having the $d$ vertices $\mathbf{x}_{\mathbf{j}}, j \neq i$, can be easily determined. Geometrically spoken, $\mathbf{x}_{\mathbf{i}}^{*}$ is the point of intersection of the $d$ Miquel spheres $S_{j}, j \neq i$, with the hyperplane containing the $(d-1)$-face of the original simplex which $\mathbf{x}_{\mathbf{i}}$ does not belong to. In this way a new $d$ simplex $S\left(\mathbf{x}_{\mathbf{0}}^{*}, \mathbf{x}_{\mathbf{1}}^{*}, \ldots, \mathbf{x}_{\mathbf{d}}^{*}\right)$ arises and relations between it and the original one for given $\lambda_{i j}$ 's could be of interest.

2. Since the matrix $\mathbf{A}_{\Lambda}$ is invertible for any $\lambda_{i j}$ the point $\mathbf{x}^{*}$ is also well-defined for $\lambda_{i j} \in$ $\{0,1\}$. For special choices of the $\lambda_{i j}$ 's we have interesting geometric intepretations, e.g. for $d=2$, letting $\lambda_{01} \rightarrow 1, \lambda_{02} \rightarrow 0$, and $\lambda_{12} \rightarrow 1$ entails that each of the limiting Miquel circles touches one side of the triangle $\triangle \mathbf{x}_{0} \mathbf{x}_{1} \mathbf{x}_{2}$ at a vertex and passes through the opposite vertex; the corresponding Miquel point turns into the Brocard point, see [1]. By means of the Theorem in Section 2 several generalizations of this point to higher dimensions are possible. In particular, for a tetrahedron $S\left(\mathbf{o}, \mathbf{x}_{1}, \mathbf{x}_{2}, \mathbf{x}_{3}\right)$ in $\mathbb{R}^{3}$ the choice $\lambda_{01}=\lambda_{02}=$ $\lambda_{03}=\lambda_{13}=1$ and $\lambda_{12}=\lambda_{23}=0$ (in the above setting) yields $\mathbf{x}^{*}$ as common point of the circumsphere $S_{0}$ and $S_{1} \cap S_{2} \cap S_{3}$, where e.g. $S_{1}$ is the unique sphere through $\mathbf{x}_{\mathbf{3}}$ touching the face triangle $\triangle \mathbf{o x}_{1} \mathbf{x}_{2}$ at $\mathbf{x}_{1}$, and $S_{2}, S_{3}$ are defined analogously.

3. The vertices of a further $d$-simplex associated with $S\left(\mathbf{o}, \mathbf{x}_{\mathbf{1}}, \ldots, \mathbf{x}_{\mathbf{d}}\right)$ and the given $\lambda_{i j}$ 's coincide with the midpoints $\mathbf{x}_{\mathbf{i}}^{\mathbf{c}}$ of the Miquel spheres $S_{i}, i=0,1, \ldots, d$. In Section 2 we have derived the following formulas:

$$
\mathbf{x}_{\mathbf{0}}^{\mathbf{c}}=\frac{1}{2}\left(\mathbf{X}^{\prime}\right)^{-1} \mathbf{x}_{\lambda} \quad \text { and } \quad \mathbf{x}_{\mathbf{i}}^{\mathbf{c}}=\frac{1}{2}\left(\mathbf{X}^{\prime}\right)^{-1} \mathbf{A}_{\Lambda} \mathbf{e}_{\mathbf{i}}+\mathbf{x}_{\mathbf{0}}^{\mathbf{c}}, \quad i=1, \ldots, d
$$

In the planar case simple geometric arguments show that the triangles $\triangle \mathbf{x}_{\mathbf{0}} \mathbf{x}_{1} \mathbf{x}_{2}$ and $\triangle \mathbf{x}_{\mathbf{0}}^{\mathbf{c}} \mathbf{x}_{\mathbf{1}}^{\mathbf{c}} \mathbf{x}_{\mathbf{2}}^{\mathbf{c}}$ are similar, cf. e.g. [1]. It is natural to ask whether the simplex $S\left(\mathbf{x}_{\mathbf{0}}^{\mathbf{c}}, \mathbf{x}_{\mathbf{1}}^{\mathbf{c}}, \ldots, \mathbf{x}_{\mathbf{d}}^{\mathbf{c}}\right)$ and the original simplex are similar for any $d \geq 2$. This fact can be expressed analytically by an orthogonal matrix $\mathbf{O}$ and some scaling factor $\gamma>0$ by requiring

$$
\mathbf{x}_{\mathbf{i}}^{\mathbf{c}}-\mathbf{x}_{\mathbf{0}}^{\mathbf{c}}=\frac{1}{2}\left(\mathbf{X}^{\prime}\right)^{-1} \mathbf{A}_{\Lambda} \mathbf{e}_{\mathbf{i}}=\gamma \mathbf{O} \mathbf{x}_{\mathbf{i}} \text { for } i=1, \ldots, d, \text { where } \gamma=\frac{1}{2}\left(\frac{\operatorname{det} \mathbf{A}_{\Lambda}}{\Delta^{2}}\right)^{1 / d} .
$$

In view of $\mathbf{O}^{\prime}=\mathbf{O}^{-1}$ and (7) these relations are equivalent to $G^{-1}(\mathbf{X})=2 \gamma^{2}\left(\mathbf{A}_{\Lambda}^{-1}+\right.$ $\left.\left(\mathbf{A}_{\Lambda}^{\prime}\right)^{-1}\right)$. This equality holds actually only for $d=2$ without additional restrictions on the $\lambda_{i j}$ 's.

4. On the other hand, for any $d \geq 2$, the particular choice $\lambda_{i j}=1 / 2,0 \leq i<j \leq d$, yields the Miquel point $\mathbf{x}^{*}=\frac{1}{2}\left(\mathbf{X}^{\prime}\right)^{-1}\left(\left\|\mathbf{x}_{1}\right\|^{2}, \ldots,\left\|\mathbf{x}_{\mathbf{d}}\right\|^{2}\right)^{\prime}$, which coincides with the circumcentre of the simplex $S\left(\mathbf{o}, \mathbf{x}_{\mathbf{1}}, \ldots, \mathbf{x}_{\mathbf{d}}\right)$. 


\section{Acknowledgements}

I wish to thank Prof. Dr. H. Martini (TU Chemnitz) who brought Konnully's paper [2] to my attention. I am grateful to Dr. L. Muche for computer simulations concerning Miquel's theorem. Many thanks also to Dr. T. Klein for his helpful comments on an earlier version of this paper and to stud. math. oec. G. Freudenreich who assisted me in the $3 \mathrm{D}$ visualization of Miquel's theorem.

\section{References}

[1] Donath, E.: Die merkwürdigen Punkte und Linien des ebenen Dreiecks. VEB Deutscher Verlag der Wissenschaften, Berlin 1968.

[2] Konnully, A.O.: Pivot theorems in $n$-space. Beiträge Algebra Geom. 10 (1980), 157-163.

[3] Miquel, A.: Mémoire de Géométrie, J. Math. Pures Appl. de Liouville 1 (1838), 485-487.

[4] Weisstein, E.W.: Miquel's Theorem. http://mathworld.wolfram.com/MiquelsTheorem.html

Lothar Heinrich

University of Augsburg

Institute of Mathematics

D-86135 Augsburg, Germany

e-mail: heinrich@math.uni-augsburg.de 